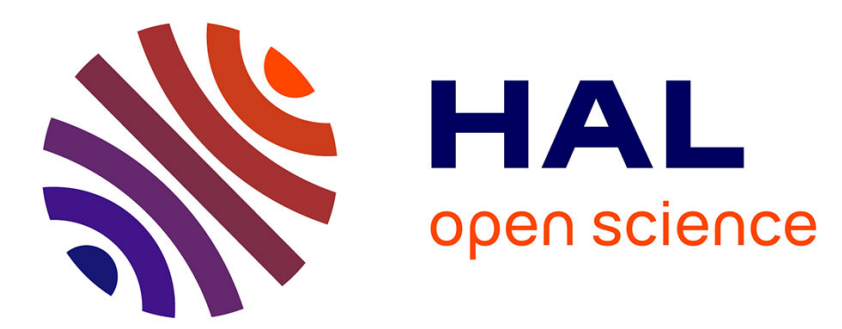

\title{
EFFECTS OF SHAPE ON UNSTABLE GROWTH OF CAVITIES INTERACTING WITH SURROUNDING SMALL VOIDS
}

Viggo Tvergaard

\section{- To cite this version:}

Viggo Tvergaard. EFFECTS OF SHAPE ON UNSTABLE GROWTH OF CAVITIES INTERACTING WITH SURROUNDING SMALL VOIDS. Philosophical Magazine, 2009, 88 (28-29), pp.33573368. 10.1080/14786430802084921 . hal-00513890

\author{
HAL Id: hal-00513890 \\ https://hal.science/hal-00513890
}

Submitted on 1 Sep 2010

HAL is a multi-disciplinary open access archive for the deposit and dissemination of scientific research documents, whether they are published or not. The documents may come from teaching and research institutions in France or abroad, or from public or private research centers.
L'archive ouverte pluridisciplinaire HAL, est destinée au dépôt et à la diffusion de documents scientifiques de niveau recherche, publiés ou non, émanant des établissements d'enseignement et de recherche français ou étrangers, des laboratoires publics ou privés. 


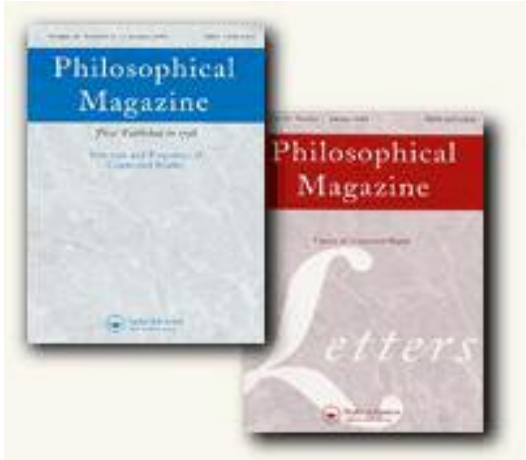

\section{EFFECTS OF SHAPE ON UNSTABLE GROWTH OF CAVITIES} INTERACTING WITH SURROUNDING SMALL VOIDS

\begin{tabular}{|r|l|}
\hline Journal: & Philosophical Magazine \& Philosophical Magazine Letters \\
\hline Manuscript ID: & TPHM-07-Nov-0320.R1 \\
\hline Journal Selection: & Philosophical Magazine \\
\hline $\begin{array}{r}\text { Date Submitted by the } \\
\text { Author: }\end{array}$ & 04-Mar-2008 \\
\hline Complete List of Authors: & Tvergaard, Viggo; Technical University of Denmark \\
\hline Keywords: & damage, plasticity \\
\hline Keywords (user supplied): & Plasticity, instability, ductile fracture. \\
\hline \multicolumn{2}{|l}{} \\
\hline
\end{tabular}

\section{ScholaronE \\ Manuscript Central}




\title{
EFFECTS OF SHAPE ON UNSTABLE GROWTH OF CAVITIES
}

\author{
INTERACTING WITH SURROUNDING SMALL \\ VOIDS
}

\author{
VIGGO TVERGAARD* \\ Department of Mechanical Engineering, Solid Mechanics \\ Technical University of Denmark, DK-2800 Kgs. Lyngby, Denmark
}

\begin{abstract}
The possibility of unstable cavity growth in a ductile metal containing many voids has been studied recently. The central spherical cavity was discretely represented, while the surrounding small-scale voids were represented by a porous ductile material model in terms of a field quantity that specifies the variation of the void volume fraction in the surrounding metal. Here the influence of the initial shape of the central void is analyzed by considering various initial spheroidal void shapes. It is shown that the evolution of the void volume fraction fields around the central void is very sensitive to the void shape. If there is no porosity in the surrounding material, the central void in a high triaxiality stress field develops towards a near spherical shape before unstable growth occurs. However, in the presence of surrounding small-scale voids the evolution of the porosity fields has much influence on the occurrence of the maximum stress point, where the central void will start to grow rapidly in interaction with fracture evolution in the surrounding material.
\end{abstract}

Keywords: Plasticity; instability; finite strains; ductile fracture.

\section{INTRODUCTION}

Cavitation instabilities in ductile metals have been found in a number of analyses for a single void far away from other voids (Bishop and Hill [1], Huang et al. [2], Tvergaard et al. [3] ). Recent studies have also considered the interaction with neighbouring voids, either represented as discrete voids of different size (Tvergaard [4]), or represented in terms of a ductile porous material model (Tvergaard and Vadillo [5]). The initial shape of the cavity analyzed has usually been taken to be spherical, but effects of initially prolate or oblate spheroidal shapes have been analysed by Tvergaard and Hutchinson [6]. 
For an elastic-plastic solid containing a single void, unstable growth occurs when the stress level in the material has reached a sufficiently high level, such that the work released in the field surrounding the expanding void is enough to drive the plastic flow associated with continued expansion. Such high stress levels are typically reached in metal-ceramic systems, where the constraint on plastic flow in the metal leads to very high stress triaxialities (e.g. see Flinn et al. [7], Ashby et al. [8], Dalgleish et al. [9]). Cavitation instabilities have also been studied in the context of nonlinear elasticity, where the instability has been interpreted either as a bifurcation from a homogeneously stressed solid to a solid containing a void, or as the growth of a pre-existing void (Ball [10], Horgan and Abeyarathne [11] and Horgan and Polignone [12]).

In the analyses of Tvergaard and Hutchinson [6] for an initially spheroidal void in an axisymmetric stress state it was found that the critical cavitation stress shows very little influence of the initial void shape. While the void volume increases by a large factor, the void shape gradually approaches that of the corresponding initially spherical void in the same axisymmetric stress state (Tvergaard et al. [3]), and therefore the critical stresses differ little. However, a non-spherical shape will affect the strain and stress fields around the growing cavity, and therefore the interaction with neighbouring small voids, if there is such interaction.

The purpose of the present paper is to extend the study of Tvergaard and Vadillo [5] to consider an initially spheroidal void, interacting with other small voids in the surrounding material. A unit cell model is analyzed, with a central spheroidal void discretely represented and with a population of small voids in the surrounding metal represented in terms of the generalized Gurson model (Gurson [13], Tvergaard [14]).

\section{PROBLEM FORMULATION}

The initial dimensions of the axisymmetric unit cell analysed are specified by the value $R_{0}$ of the outer radius and the height $R_{0}$, in addition to the initial half-axes $a_{0}$ and $b_{0}$ of the void in the radial and axial directions, respectively (Fig. 1). In the cylindrical reference coordinate system $x^{1}$ is the axial coordinate, $x^{2}$ the radial coordinate and $x^{3}$ the circumferential angle. Finite strains are accounted for, and the boundary conditions are

$\dot{u}^{1}=0, \quad \dot{T}^{2}=\dot{T}^{3}=0, \quad$ at $x^{1}=0$

$\dot{u}^{1}=\dot{U}_{I}, \quad \dot{T}^{2}=\dot{T}^{3}=0, \quad$ at $\quad x^{1}=R_{0}$

$\dot{u}^{2}=\dot{U}_{I I}, \quad \dot{T}^{1}=\dot{T}^{3}=0, \quad$ at $x^{2}=R_{0}$

$\dot{T}^{i}=0, \quad$ at $\quad\left(x^{1} / b_{0}\right)^{2}+\left(x^{2} / a_{0}\right)^{2}=1$

The two constants $\dot{U}_{I}$ and $\dot{U}_{I I}$ are displacement increments and the ratio $\dot{U}_{I I} / \dot{U}_{I}$ is calculated in each increment such that there is a fixed prescribed ratio $\rho=T / S$, between the macroscopic true stresses [15], where $S$ is the average true stress in the axial direction, and $T$ is the average true stress in the radial direction.

Finite strains are accounted for, using a Lagrangian convected coordinate formulation of the field equations. A material point is identified in the reference configuration by $x^{i}$, and the contravariant components of the Cauchy stress tensor $\sigma^{i j}$ and the Kirchhoff stress 
tensor $\tau^{i j}$ are related by $\tau^{i j}=\sqrt{G / g} \sigma^{i j}$. The metric tensors in the current and the reference configuration are denoted by $G_{i j}$ and $g_{i j}$, with the determinants $G$ and $g$, respectively.

The generalized Gurson model $[13,14]$ has an approximate yield condition of the form $\Phi\left(\sigma^{i j}, \sigma_{M}, f\right)=0$, where $\sigma^{i j}$ is the average macroscopic Cauchy stress tensor, $\sigma_{M}$ is an equivalent tensile flow stress representing the actual microscopic stress-state in the matrix material and $f$ is the current void volume fraction. The yield condition is

$\Phi=\frac{\sigma_{e}^{2}}{\sigma_{M}^{2}}+2 q_{1} f^{*} \cosh \left(\frac{q_{2}}{2} \frac{\sigma_{k}^{k}}{\sigma_{M}}\right)-\left[1+\left(q_{1} f^{*}\right)^{2}\right]=0$

where $\sigma_{e}=\left(3 s_{i j} s^{i j} / 2\right)^{1 / 2}$ is the macroscopic effective Mises stress, $s^{i j}=\sigma^{i j}-G^{i j} \sigma_{k}^{k} / 3$ is the stress deviator, and $q_{1}=q_{2}=1$ according to [18]. This approximation can be improved, as suggested in $[15,16]$, by using the values $q_{1}=1.5$ and $q_{2}=1$. The function $f^{*}(f)$ was introduced [17] to model the effect of void coalescence

$f^{*}(f)= \begin{cases}f & , \text { for } f \leq f_{C} \\ f_{C}+\frac{f_{U}^{*}-f_{C}}{f_{F}-f_{C}}\left(f-f_{C}\right) & , \text { for } f>f_{C}\end{cases}$

where $f_{U}^{*}=f^{*}\left(f_{F}\right)$ with the value $f_{U}^{*}=1 / q_{1}$. The parameter values chosen for $(6)$ are $f_{C}=0.15$ and $f_{F}=0.25$.

The rate of equivalent plastic work in the matrix material is assumed equal to the macroscopic plastic work

$\sigma^{i j} \dot{\eta}_{i j}{ }^{P}=(1-f) \sigma_{M} \dot{\varepsilon}_{M}^{P}$

where $\dot{\eta}_{i j}{ }^{P}$ is the plastic part of the macroscopic strain rate and

$\dot{\varepsilon}_{M}^{P}=\left(\frac{1}{E_{t}}-\frac{1}{E}\right) \dot{\sigma}_{M}$

in terms of Young's modulus $E$ and the tangent modulus $E_{t}$ for the uniaxial true stressnatural strain curve at the stress level $\sigma_{M}$. This curve is represented by a piecewise power law

$\varepsilon= \begin{cases}\frac{\sigma}{E} & \sigma \leq \sigma_{Y} \\ \frac{\sigma_{Y}}{E}\left(\frac{\sigma}{\sigma_{Y}}\right)^{1 / N} & \sigma>\sigma_{Y}\end{cases}$

where $\sigma_{Y}$ and $N$ are the yield stress and the strain hardening exponent, respectively.

The change of the void volume fraction during an increment of deformation is taken to be

$\dot{f}=(1-f) G^{i j} \dot{\eta}_{i j}^{P}+\mathrm{A} \dot{\sigma}_{M}+\mathrm{B}\left(\sigma_{k}^{k}\right)^{\bullet} / 3$

For strain controlled nucleation, Chu and Needleman [18] have proposed the following expressions for the nucleation coefficients A and B

$$
\mathrm{A}=\left(\frac{1}{E_{t}}-\frac{1}{E}\right) \frac{f_{N}}{s \sqrt{2 \pi}} \exp \left[-\frac{1}{2}\left(\frac{\varepsilon_{M}^{P}-\varepsilon_{N}}{s}\right)^{2}\right] \quad, \quad \mathrm{B}=0
$$


corresponding to a normal distribution, where $\varepsilon_{N}$ is the mean strain for nucleation, $s$ is the standard deviation, and $f_{N}$ is the volume fraction of void nucleating particles.

The plastic part of the strain-rate is taken to be

$\dot{\eta}_{i j}^{P}=\frac{1}{H} m_{i j}^{G} m_{k l}^{F} \stackrel{\nabla}{\sigma}{ }^{k l}$

where ${ }^{\nabla} \sigma^{k l}$ is the Jaumann rate of the Cauchy stress, the expressions for $H$ and the tensors $m_{i j}^{G}$ and $m_{i j}^{F}$ are given in [14]. Plastic yielding initiates when $\Phi=0$ and $\dot{\Phi}>0$ during elastic deformation, while continued plastic loading requires $\Phi=0$ and $m_{k l}^{F} \sigma^{k l} / H \geq 0$. The incremental stress-strain relationship is of the form $\dot{\tau}^{i j}=L^{i j k l} \dot{\eta}_{k l}$, where $L^{i j k l}$ are the instantaneous moduli.

The analyses in [5] accounted for the dependence of elastic properties on porosity, by using expressions derived by Tandon and Weng [19], but it was found that this reduced elastic stiffness had negligible effect. Very similar results were obtained by using linear interpolations of the expressions for $E(f)$ and $v(f)$, as suggested by Fleck et al. [20], taking $E(f)=0$ when $f=1 / q_{1}$. These latter expressions are used in the present computations.

The numerical method applied is based on the incremental principle of virtual work, in which the displacement fields are approximated in terms of axisymmetric triangular elements, arranged in quadrilaterals of crossed triangles. Fig. 1 shows an example of the initial mesh around an oblate spheroidal void with $a_{0} / b_{0}=2$. A special Rayleigh-Ritz finite element method [21] is used to improve the numerical stability when the occurrence of a cavitation instability is close. This makes it possible to prescribe node displacements on the void surface or inside the mesh without applying a load, rather than prescribing the end displacement of the unit cell. According to (6), final ductile failure occurs when $f$ reaches $f_{F}$. In the present studies, as in [5], final failure is represented in terms of a small yield surface technique, in which expression (10) is used until $f^{*}(f)=0.99 f_{U}^{*}$, and after that the value of $f$ is kept constant.

\section{RESULTS}

For a spherical void the ratio of the initial void radius to the outer radius of the cell model is taken to be $a_{0} / R_{0}=1 / 3000$, so that the void volume fraction corresponding to the central void is very small, $2.5 \times 10^{-11}$. When the central void is spheroidal, the initial half-axes $a_{0}$ and $b_{0}$ are chosen such that this void volume fraction is the same. The values of the material parameters are taken to be $\sigma_{Y} / E=0.003$ and $v=0.3$, with the power hardening exponent $N=0.1$ in most of the cases. The value of the initial void volume fraction, $f_{0}$, or of the nucleation parameters, will be different for each case analysed. It is noted that even though the central void is so small relative to the cell analyzed that it represents a very small void volume fraction, rather large values of $f_{0}$ for the surrounding matrix material can still 
represent a population of voids in which each of the voids is much smaller than the central void.

In [5] computations for $f_{0}=0.1$, with the macroscopic stress ratio $\rho=T / S=0.9$ and $N=0.1$, were used to show that even for this high initial porosity the stress level is only slightly affected. This is surprising, and therefore the result is considered in more detail here. According to the expressions of Fleck et al. [20] the porosity reduces the elastic material parameters to $0.85 E$ and $0.85 \mathrm{v}$, but the maximum stress levels are only reduced by $1.6 \%$ while the corresponding strain levels are increased by $3 \%$ and $4 \%$, respectively, compared to the result when elasticity is independent of voids. However, full plastic yielding of the cell model has occurred long before the maximum stress is reached, and therefore the small changes of the stress and strain levels at the maximum are heavily dominated by plasticity effects. A similar comparison for $f_{0}=0.01$ has shown a stress reduction of only $0.2 \%$, so the reduced elastic stiffness due to porosity has a very small effect.

The effect of initially spheroidal void shapes is analyzed here for the stress ratio $\rho=0.9$ and strain hardening exponent $N=0.1$. The analyses consider oblate spheroids with $a_{0} / b_{0}=4$ or $a_{0} / b_{0}=2$, prolate spheroids with $a_{0} / b_{0}=0.5$ or $a_{0} / b_{0}=0.25$, and spherical voids $\left(a_{0} / b_{0}=1\right)$. Fig. 2 shows curves calculated for $f \equiv 0$. Here the curves for the spherical void have been continued up to $V / V_{o}=41.1$, where the stress level is $S / \sigma_{Y}=5.61$, while the other four computations are stopped earlier, due to numerical instabilities. Fig. $2 b$ confirms the result found by Tvergaard and Hutchinson [6] that the ratio $a / b$ develops towards unity, indicating that all the growing voids are going to become nearly spherical. The corresponding development of the stress level vs. void volume in Fig. $2 b$ shows very little difference between the curves for the five cases.

Fig. 3 shows results of computations similar to to those in Fig. 2, but now there is an initial void volume fraction $f_{0}=0.01$ in the material surrounding the central void. At this rather large void volume fraction in the matrix material the unit cell becomes fully plastic at an early stage, as was also found above for $f_{0}=0.1$, and elastic unloading starts to occur later when coalescence of the small-scale voids initiates around the central void, in a toroidal region near the symmetry plane $x^{1}=0$. The evolution of $a / b$ in Fig. $3 b$ is initially similar to that in Fig. $2 \mathrm{~b}$, but after the initiation of coalescence the ratio $a / b$ tends towards a value around 0.5 rather than the value 1.0. Fig. 3a shows very little difference between the stress levels reached in the five different cases.

The curves in Fig. $3 b$ show the current ratio $a / b$ defined by all elements, including the damaged elements where the yield surface is very small $\left(f^{*}=0.99 f_{U}^{*}\right)$. If the current hole dimensions should include the crack growing from the central void by void coalescence in the surrounding material, the development of the curves in Fig. $3 \mathrm{~b}$ would be different.

The initial void volume fraction $f_{o}=0.001$ in Fig. 4 is small enough so that most of the unit cell remains elastic for the stress state considered $(\rho=0.9)$ and plasticity is limited to a rather small region around the central void. Here, the stress evolution in Fig. 4 shows a clear difference between the curves for the the five different initial geometries of the central voids analysed. As was discussed in [5] for the initially spherical void, the rapid load drop at $V / V_{o} \approx 1.60$ occurs when the void volume fraction $f$ grows rather suddenly in a toroidal region at the symmetry plane $x^{1}=0$, at a distance of about $4.5 a_{0}$ outside the current surface of the central void. Fig. 4 shows that similar sudden load drops occur for all five initial geometries of the central void. The stress value just before this sudden load drop occurs 
is also the maximum stress reached in each case, and Fig. 4 shows that the maximum stresses reached in the five cases are nearly identical. The peak occurs first for the spherical void and last for the voids with the largest aspect ratios, $a / b=0.25$ and $a / b=4$.

The mechanisms behind these differences in the occurrence of the stress peaks in Fig. 4 can be understood from the contours of constant void volume fraction in Fig. 5, soon after the sudden load drop. For $a / b=0.25$ at $V / V_{o}=2.02$, Fig. 5a shows that rapid void growth has occurred in a toroidal region well away from the central void at the symmetry plane $x^{1}=0$, while for $a / b=4$ at $V / V_{o}=2.02$ Fig. $5 b$ shows that rapid void growth has started from the sharp end of the surface of the central void. In Fig. 5b the voids grow most rapidly where the strains are largest, even though the hydrostatic tension is rather low at the surface of the central void, and thus the behaviour at the oblate spheroid approaches that for a pennyshaped crack. In Fig. 5a for the prolate spheroid the minimum surface curvature at the side of the central void gives rather low strains, so here the higher hydrostatic tension at some distance from the void surface has the dominant effect on where the voids start to grow most rapidly. The behaviour in Fig. 5a is similar to that found for the spherical void, but void growth develops more slowly due to the spheroidal shape, and therefore the stress peak in Fig. 4 occurs later. Also in the case of Fig. 5b, where the mechanism is strain dominated, the peak occurs later. It is noted that Fig. 5a also shows some damage at the highly curved end of the central void, but this damage evolution has stopped and does not grow any further in the range considered.

For each of the five cases in Fig. 4 the small scale voids at the plane $x^{1}=0$ grow to coalescence, so that subsequently a crack develops along this plane. In the case of Fig. 5b the crack grows from the surface of the central void into the material, through the region of slightly increased porosity shown in the figure at some distance from the central void. In Fig. $5 \mathrm{a}$ the crack initiates at some distance from the central void and subsequently grows in both directions, most rapidly towards the central void, but at the end of the corresponding curve in Fig. 4 the crack has not yet reached the central void surface. After the rapid load drops in Fig. 4 the stress levels decay more slowly, as damage evolves, but for still increasing $V / V_{o}$ the predictions will gradually lose accuracy, as the cracks grow into cruder mesh regions.

When a cavitation instability occurs in an elastic-plastic material with no damage the remote stresses and strains remain constant, while growth of the cavity is driven by the elastic energy stored in the material volume that is huge relative to the cavity [2,3]. In the cases of Fig. 4, where damage plays a role, the instability occurs at the load maximum since the behaviour is controlled by the remote load. Even though the numerical procedure has been able to follow an unstable equilibrium path, the subsequent stress levels lower than the maximum in Fig. 4 mean that the cavity growth and the crack growth in the symmetry plane $\left(x^{1}=0\right)$ will occur dynamically, driven by the stored elastic energy. When the curves in Fig. 4 start to show slowly growing stress levels at $V / V_{o} \approx 3$ this is expected to be a mesh effect which might be avoided if a uniform mesh had been applied.

Fig. 6 shows similar curves as those in Fig. 4, but now for $f_{o}=0.0001$. As in Fig. 4 a sudden load drop occurs when the porosity evolution starts to dominate the behaviour, and again the spherical void shows the earliest load drop, while this occurs last for the voids with the highest aspect ratios, 0.25 or 4 . The reductions of the stress levels occur more abruptly here than in Fig. 4, and the reductions are larger. While in [5], for the spherical central void, it was not possible to numerically follow this abrupt stress drop, it has been possible here by more extensive use of the special Rayleigh-Ritz finite element method [21] to prescribe node displacements inside the mesh without applying a load. 
The curves in Fig. 7 are calculated for the very small initial porosity $f_{o}=10^{-5}$. As expected the rapid stress reductions here occur later and are larger than found in Fig. 6. This is seen for the two curves where it has been possible to continue the computations beyond this stage. The other three curves clearly mark the initiation of the rapid stress drop, again so that the load drop occurs last for the voids with the highest aspect ratios. The evolution of $a / b$ in Fig. 7b is very similar to that in Fig. 2b until the rapid load reductions occur (Fig. 7a).

A different type of material behaviour is considered in Fig. 8. Here, the initial void volume fraction is zero, $f_{o}=0$, but voids nucleate according to a strain controlled rule (11), with the volume fraction of void nucleating particles $f_{N}=0.03$, the mean nucleation strain $\varepsilon_{N}=0.2$ and the standard deviation $s=0.1$. Here, the void volume fraction remains zero everywhere, except for a small region in the near vicinity of the central void, where nucleated voids develop into a crack that grows from the surface of the central void along the symmetry plane $x^{1}=0$. As in the previous figures, a sudden load drop occurs first for the spherical void, at a volume increase somewhat larger than found before. For the void with aspect ratio 0.5 the load drop is a little delayed, while for the aspect ratio 0.25 the delay is much larger. For the oblate spheroid with $a_{0} / b_{0}=2$ the sudden load drop occurs a great deal later than found for $a_{0} / b_{0}=0.5$, and for the larger aspect ratio 4 no sudden load drop is found in the range analysed. Fig. 8 shows that after the sudden load drop the value of $S / \sigma_{Y}$ decays a great deal, but afterwards the value starts to increase again, more slowly. This slow increase of the stress level occurs while the crack grows into a region with cruder and cruder mesh, and it is considered to be mainly a mesh effect.

\section{DISCUSSION}

Structural metals with two populations of void nucleating particles or pre-existing voids have been modelled previously [22] by using a discrete representation of the larger voids, while the small-scale voids in the surrounding material are represented in terms of a porous ductile material model. The same type of idea has been used in analyses of crack growth in ductile materials (e.g. [23]) and in a model that accounts for coupling between growth of big voids and coalescence of smaller surrounding voids [24]. In [5] such a material with a central spherical void was subjected to stress states with so high triaxiality that a cavitation instability could be expected, and the effect of damage by coalescence of the surrounding small scale voids was studied. In the present investigation it has been found that the influence of the surrounding small scale voids shows significant sensitivity to the shape of the larger central void, as the stress- and strain concentrations that control the porosity evolution depend on the the central void shape.

In [5] the effect of porosity on elastic properties was incorporated in the porous ductile material model, and curves were shown to illustrate that this had very little influence on the predictions, even for a void volume fraction as high as $f_{0}=0.1$. This is somewhat surprising, since the unstable growth occurs when the stress level in the material has reached a level, at which the work released in the field surrounding the expanding void is enough to drive the plastic flow associated with continued expansion, and the stored elastic energy is reduced when Young's modulus is reduced by a factor 0.85 . Here, further studies of this point have revealed that for $f_{0}=0.1$ full plastic yielding of the cell model occurs long 
before the maximum stress is reached, and therefore the effect of the smaller elastic energy density is less visible. Even for $f_{0}=0.01$ the cell model is fully plastic before the maximum stress is reached.

The cell model analyses here show that in all cases a sudden load drop occurs when void growth and coalescence starts to develop rapidly in the surrounding material, as was found in [5] for a spherical central void. For prolate spheroids, with $a_{0} / b_{0}<1$, rapid void growth occurs in a toroidal region well away from the central void at the symmetry plane $x^{1}=0$, while for oblate spheroids, with $a_{0} / b_{0}>1$, the rapid void growth starts from the sharp end of the surface of the central void, so that the behaviour at oblate spheroids approaches that for a penny-shaped crack. For the prolate spheroid the rather low surface curvature at the side of the central void gives lower strains, and therefore the high hydrostatic tension at some distance from the void surface dominates. Both competing mechanisms play a role for all the spheroidal void shapes and the present results show that for each value of $f_{0}$ the sudden load drop occurs earlier for the spherical central void than for oblate or prolate voids considered.

When the crack grows into the porous ductile material there is the possibility that shear bands could form, but in the absence of soft spots to attract the crack path it has been found [23] that cracks prefer to grow on the initial crack plane. Growth along shear bands has been predicted for the penny-shaped crack that forms in the centre of the neck of a round tensile bar [17], but this involves interaction with the free surface of the bar. In any case, prediction of shear bands would be mesh sensitive.

Very low levels of initial porosity in the surrounding material are considered here, as low as $f_{o}=10^{-5}$ in Fig. 7, but the behaviour found is rather similar to that for a hundred times higher initial porosity, with the difference that the sudden load drops are delayed to a higher value of $V / V_{o}$. In the case of strain controlled nucleation with no initial porosity the results found are also a sudden load drop at a high value of $V / V_{o}$.

The focus here is on the effect of different spheroidal void shapes, and therefore the studies are limited to a special level of high stress triaxiality, $\rho=0.9$, and a special value of the strain hardening exponent, $N=0.1$. However, for the spherical shape of the central void the studies have been extended to different values of $\rho$, and to different hardening levels [5].

\section{REFERENCES}

[1]R.F. Bishop, R. Hill and N.F. Mott, The theory of indentation and hardness tests. Proc. Phys. Sco. 57147 (1945).

[2]Y. Huang, J.W. Hutchinson and V. Tvergaard, Cavitation instabilities in elastic-plastic solids. J. Mech. Phys. Solids 39223 (1991).

[3]V. Tvergaard, Y. Huang and J.W. Hutchinson, Cavitation instabilities in a power hardening elastic-plastic solid. Eur. J. Mech. A/Solids 11215 (1992). 
[4]V. T vergaard, Interaction of very small voids with larger voids. Int. J. Solids Structures 353989 (1998).

[5]V. Tvergaard and G. Vadillo, Influence of porosity on cavitation instability predictions for elastic-plastic solids. Int. J. Mech. Sci. 49210 (2007).

[6]V. Tvergaard and J.W. Hutchinson, Effect of initial void shape on the occurrence of cavitation instabilities in elastic-plastic solids. J. of Appl. Mech. 60807 (1993).

[7]B. Flinn, M. Rühle and A.G. Evans, Toughening in composites of $\mathrm{Al}_{2} \mathrm{O}_{3}$ reinforced with Al. University of California, Santa Barbara, Materials Department Report

(1989).

[8]M.F. Ashby, F.J. Blunt and M. Bannister, Flow characteristics of highly constrained metal wires. Acta Metall. 371847 (1989).

[9]B.J. Dalgleish, K.P. Trumble and A.G. Evans, The strength and fracture of alumina bonded with aluminum alloys. Acta Metall. 371923 (1989).

[10] J.M. Ball, Discontinuous equilibrium solutions and cavitation in nonlinear elasticity. Phil. Trans. R. Soc. London A306 557 (1982).

[11] C.O. Horgan and R. Abeyaratne, A bifurcation problem for a compressible nonlinearly elastic medium: Growth of a microvoid. J. Elasticity 16189 (1986).

[12] C.O. Horgan and D.A. Polignone, Cavitation in nonlinearly elastic solids: A review. Appl. Mech. Rev. 48471 (1995).

[13] A.L. Gurson, Continuum theory of ductile rupture by void nucleation and growth - I. Yield criteria and flow rules for porous ductile media. J. Engng. Materials Technol. 99 2 (1977).

[14] V. Tvergaard, Material failure by void growth to coalescence. Advances in Applied Mechanics 2783 (1990).

[15] V. Tvergaard, On localization in ductile materials containing spherical voids. Int. J. Fracture 18237 (1982).

[16] V. Tvergaard, Influence of voids on shear band instabilities under plane strain conditions. Int. J. Fracture 17389 (1981).

[17] V. Tvergaard and A. Needleman, Analysis of the cup-cone fracture in a round tensile bar. Acta Metall. 32157 (1984).

[18] C.C. Chu and A. Needleman, Void nucleation effects in biaxially stretched sheets. J. Eng. Materials Technol. 102249 (1980).

[19] G.P. Tandon and G.J. Weng, A Theory of particle-reinforced plasticity. Transactions of the ASME, J. of Applied Mechanics 55:126-135 (1988). 
[20] N.A. Fleck, H. Otoyo and A. Needleman, Indentation of porous solids. Int. J. Solids Structures 291613 (1992).

[21] V. Tvergaard, Effect of thickness inhomogeneities in internally pressurized elasticplastic spherical shells. J. Mech. Phys. Solids 24291 (1976).

[22] V. Tvergaard, Ductile fracture by cavity nucleation between larger voids. J. Mech. Phys. Solids 30265 (1982).

[23] V. Tvergaard and A. Needleman, Effect of crack meandering on dynamic, ductile fracture. J. Mech. Phys. Solids 40447 (1992).

[24] K. Enakoutsa, J.-B. Leblond and B. Audoly, Influence of continuous nucleation of secondary voids upon growth and coalescence of cavities in porous ductile metals.

Proceedings of ICF11, Turin, Italy, 20-25 March 2005. On CD. 


\section{Figure captions:}

Fig. 1. Mesh near the central void, used for numerical analyses with $a_{0} / b_{0}=2$.

Fig. 2. Influence of various initially spheroidal void shapes for $f \equiv 0, \rho=0.9$ and $N=0.1$. (a) Stress vs. volume. (b) Evolution of void aspect ratio.

Fig. 3. Influence of various initially spheroidal void shapes for $f_{o}=0.01, \rho=0.9$ and $N=0.1$, when there is no void nucleation. (a) Stress vs. volume. (b) Evolution of void aspect ratio.

Fig. 4. Influence of various initially spheroidal void shapes for $f_{o}=0.001, \rho=0.9$ and $N=0.1$, when there is no void nucleation.

Fig. 5. Contours of constant void volume fraction at the $V / V_{o}=2.02$, for computations with $f_{o}=0.001$, $\rho=0.9$ and $N=0.1$. (a) For $a_{0} / b_{0}=0.25$. (b) For $a_{0} / b_{0}=4$.

Fig. 6. Influence of various initially spheroidal void shapes for $f_{o}=0.0001, \rho=0.9$ and $N=0.1$, when there is no void nucleation.

Fig. 7. Influence of various initially spheroidal void shapes for $f_{o}=10^{-5}, \rho=0.9$ and $N=0.1$, when there is no void nucleation. (a) Stress vs. volume. (b) Evolution of void aspect ratio.

Fig. 8. Influence of plastic strain controlled nucleation according to (2.11), for $f_{o}=0.0, f_{N}=0.03$, $\varepsilon_{N}=0.2$ and $s=0.1$, with $\rho=0.9$ and $N=0.1$. 


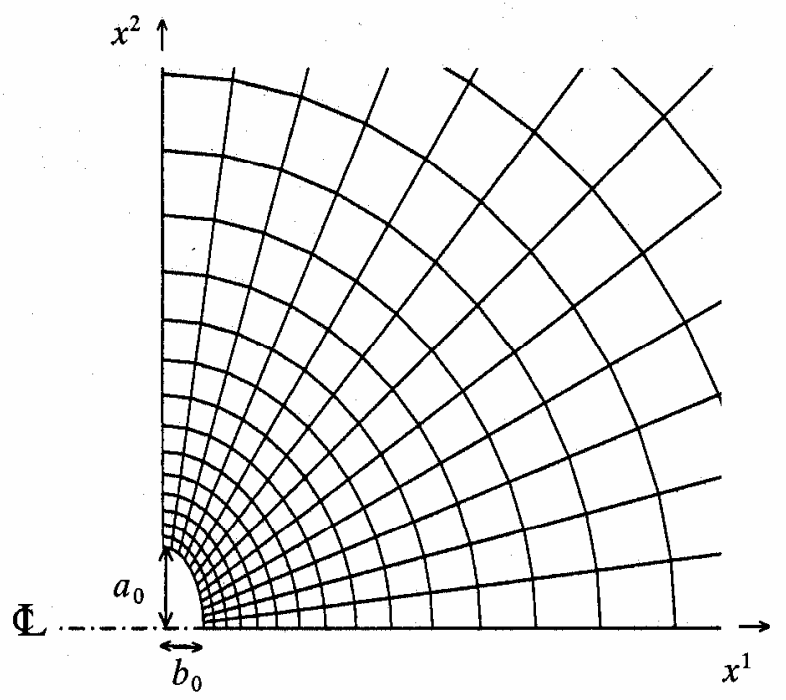

Fig. 1. Mesh near the central void, used for numerical analyses with $a_{0} / b_{0}=2$.
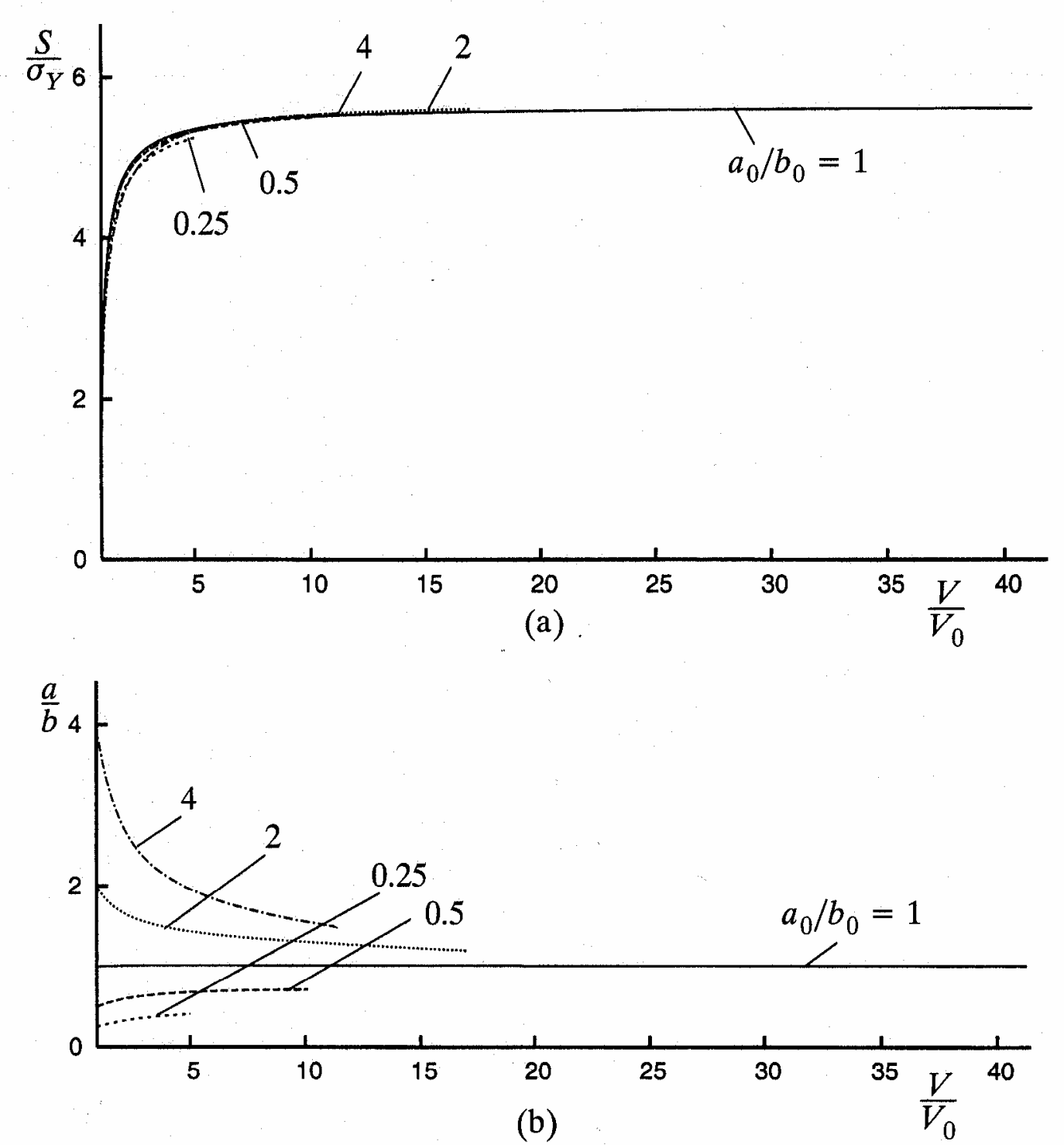
Fig. 2. Influence of various initially spheroidal void shapes for $f \equiv 0, \rho=0.9$ and $N=0.1$. (a) Stress vs. volume. (b) Evolution of void aspect ratio.
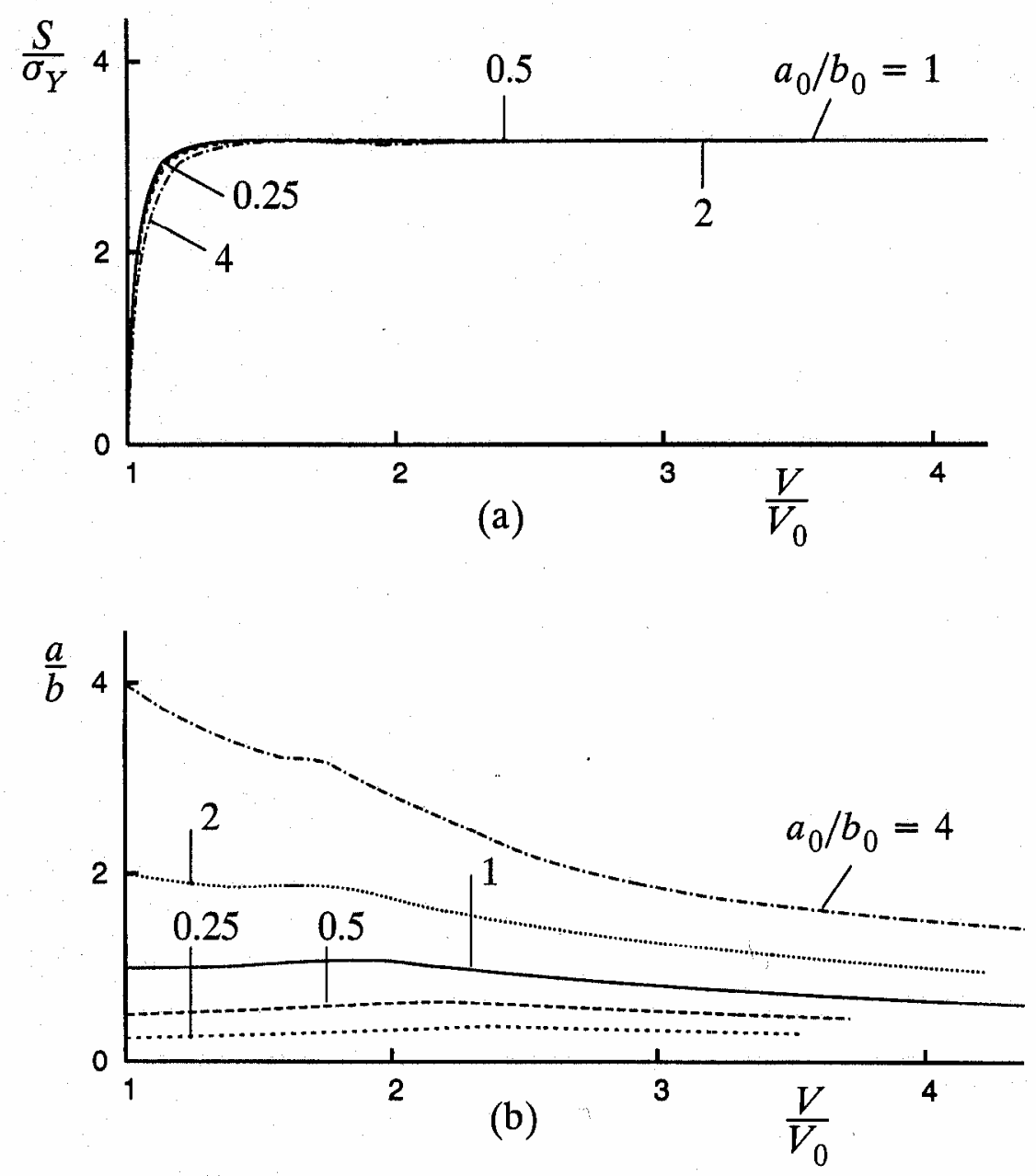

Fig. 3. Influence of various initially spheroidal void shapes for $f_{o}=0.01, \rho=0.9$ and $N=0.1$, when there is no void nucleation. (a) Stress vs. volume. (b) Evolution of void aspect ratio. 


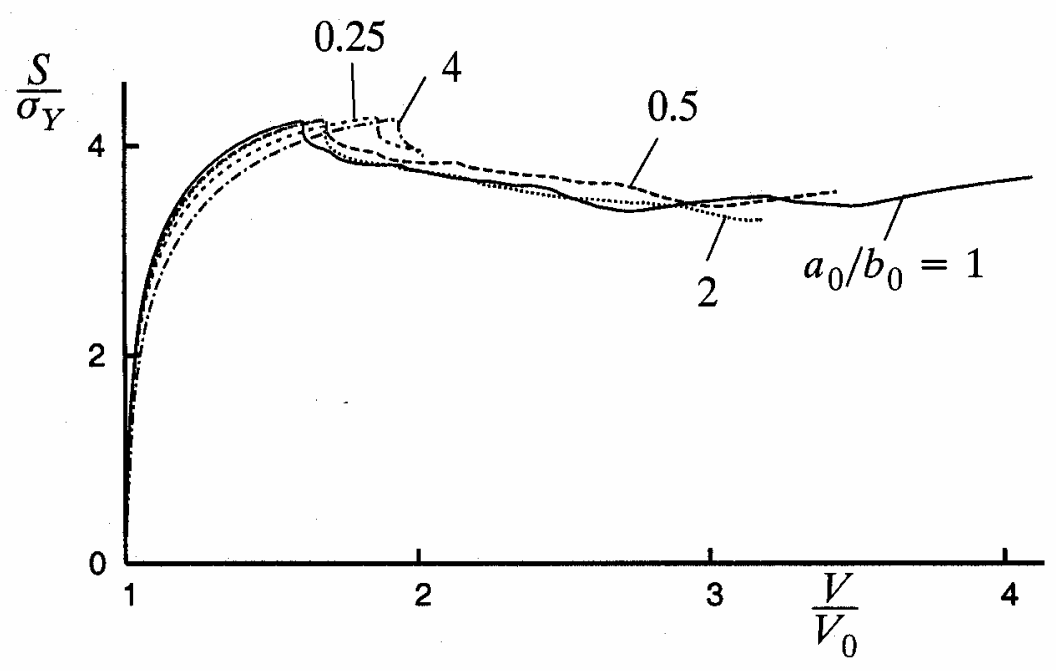

Fig. 4. Influence of various initially spheroidal void shapes for $f_{o}=0.001, \rho=0.9$ and $N=0.1$, when there is no void nucleation.
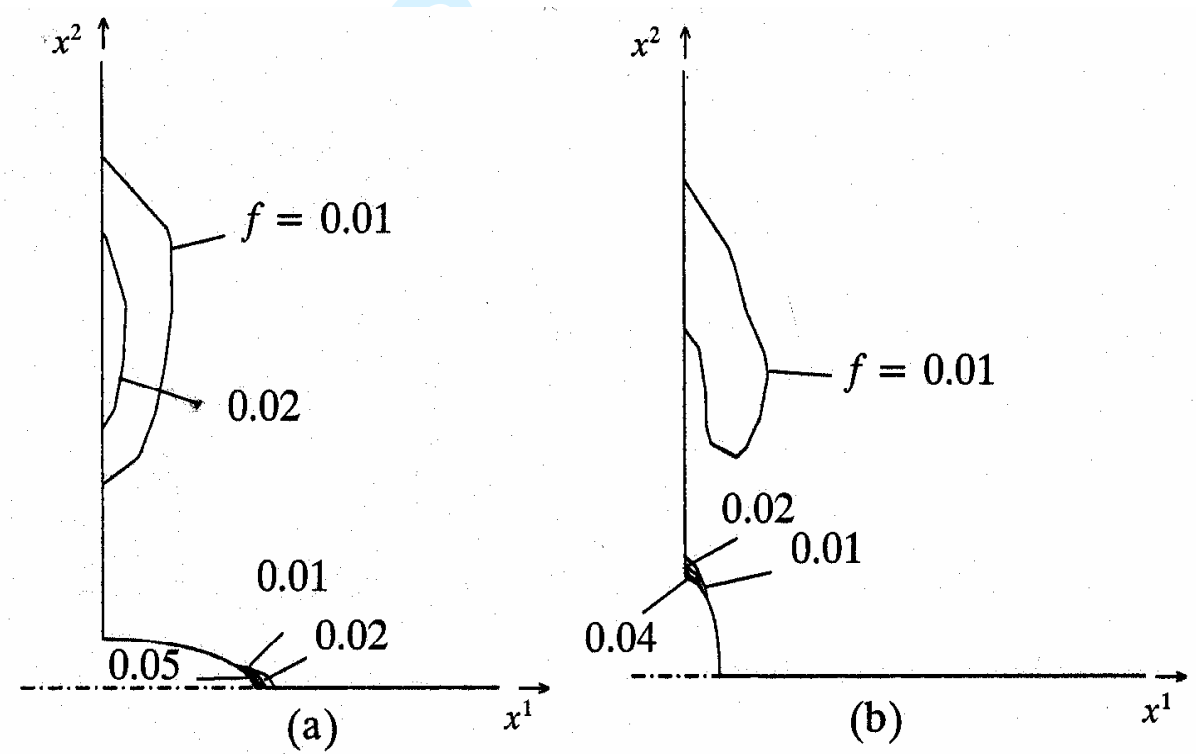

Fig. 5. Contours of constant void volume fraction at the $V / V_{o}=2.02$, for computations with $f_{o}=0.001$, $\rho=0.9$ and $N=0.1$. (a) For $a_{0} / b_{0}=0.25$. (b) For $a_{0} / b_{0}=4$. 


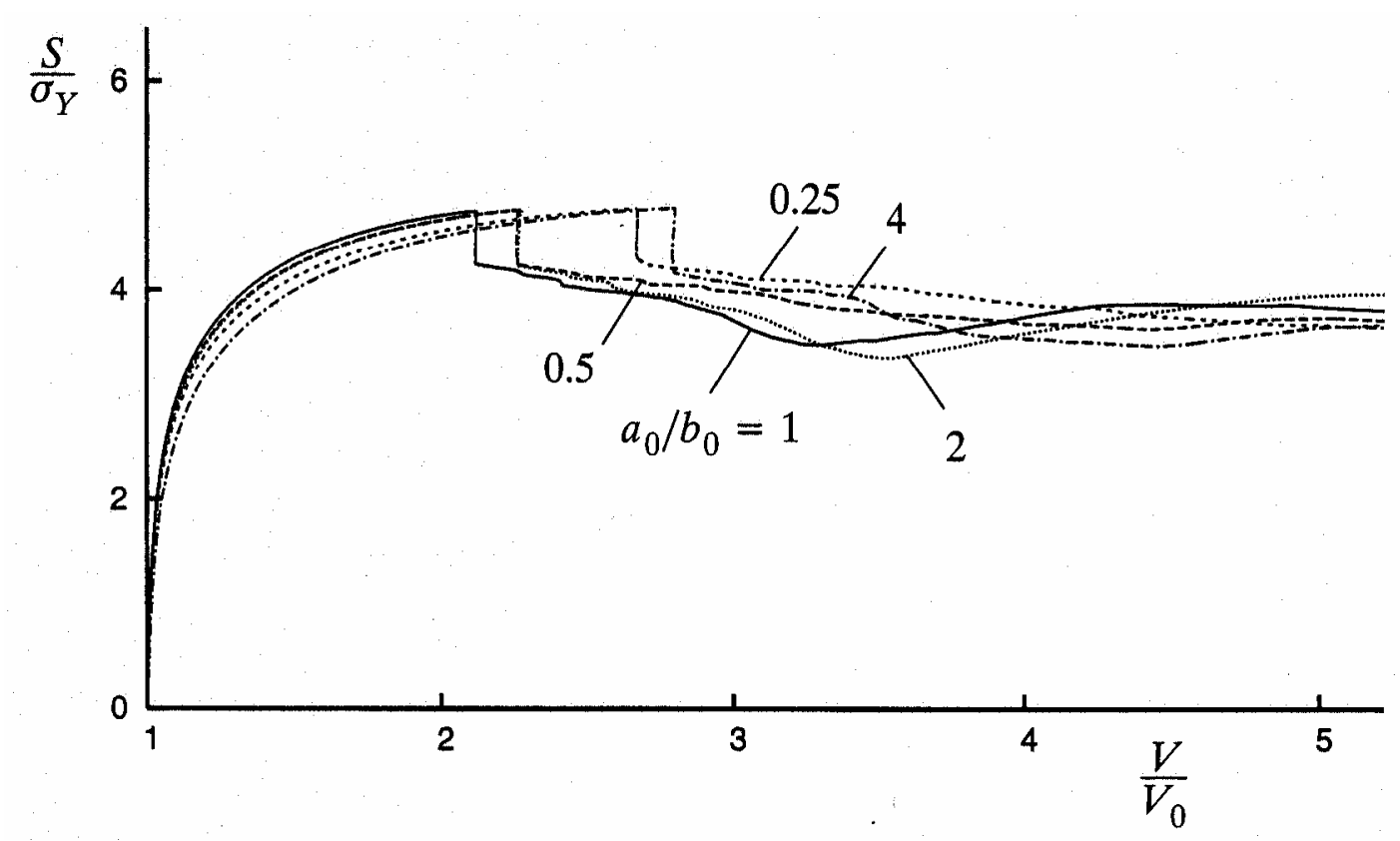

Fig. 6. Influence of various initially spheroidal void shapes for $f_{o}=0.0001, \rho=0.9$ and $N=0.1$, when there is no void nucleation. 

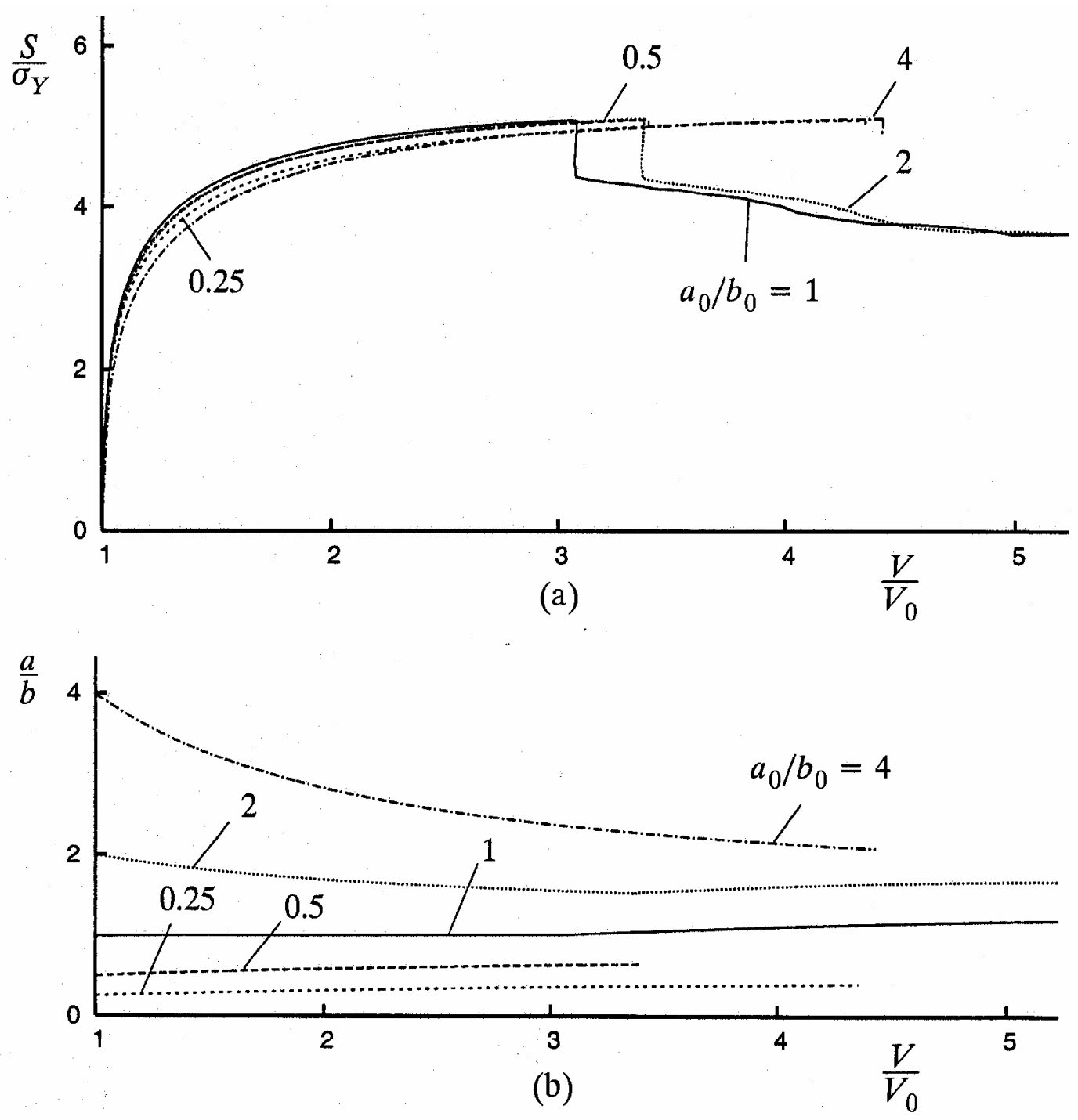

Fig. 7. Influence of various initially spheroidal void shapes for $f_{o}=10^{-5}, \rho=0.9$ and $N=0.1$, when there is no void nucleation. (a) Stress vs. volume. (b) Evolution of void aspect ratio. 


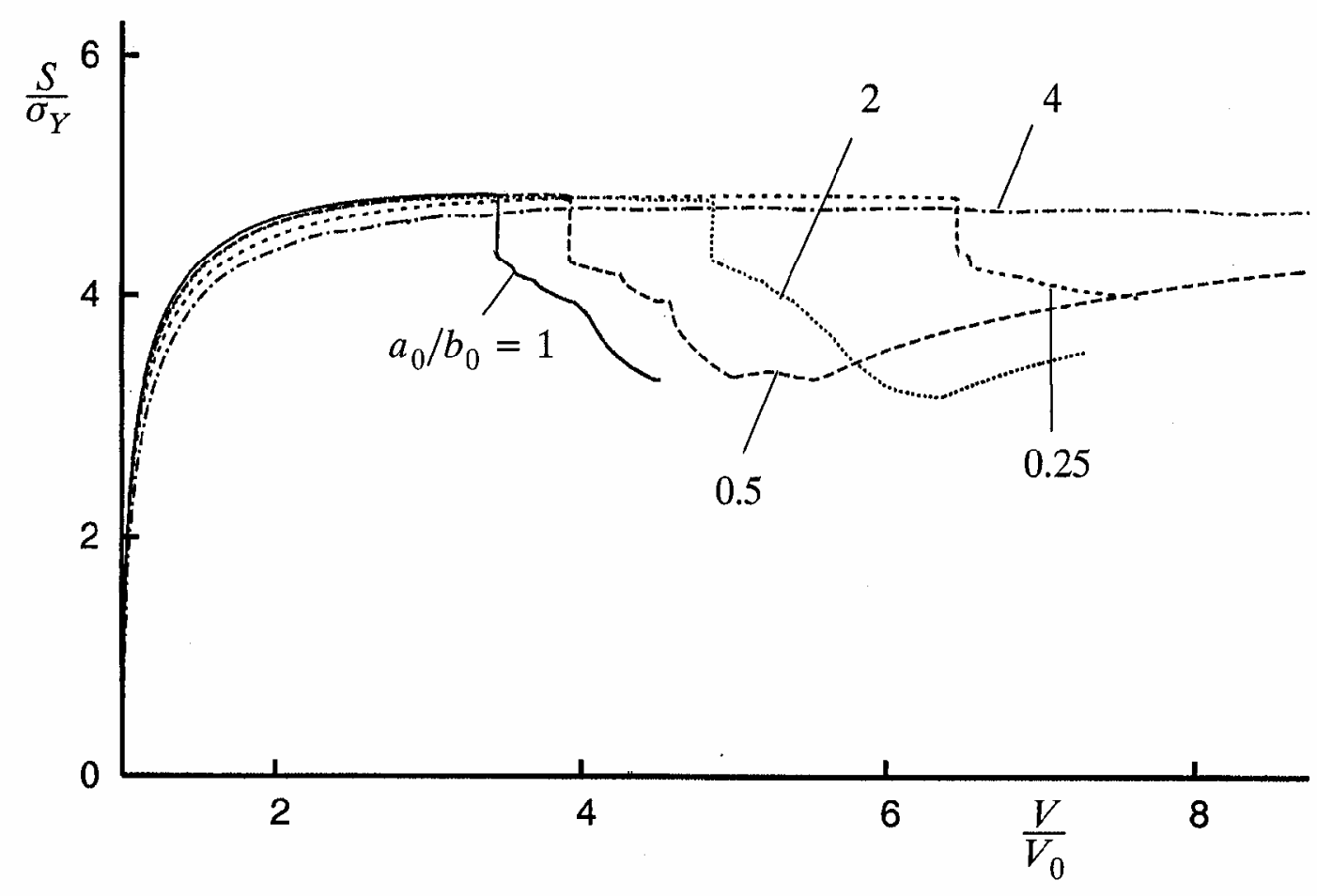

Fig. 8. Influence of plastic strain controlled nucleation according to (2.11), for $f_{o}=0.0, f_{N}=0.03$, $\varepsilon_{N}=0.2$ and $s=0.1$, with $\rho=0.9$ and $N=0.1$. 